\title{
Does the patellofemoral joint need articular cartilage?
}

\author{
Lars Blønd $^{1} \cdot$ Simon Donell ${ }^{2}$
}

Published online: 3 September 2015

(C) European Society of Sports Traumatology, Knee Surgery, Arthroscopy (ESSKA) 2015

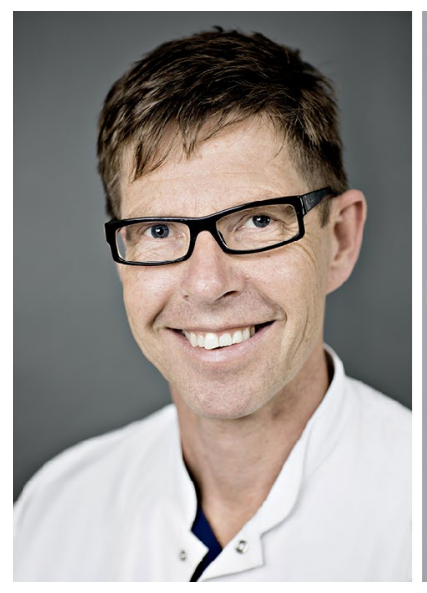

Lars Blond

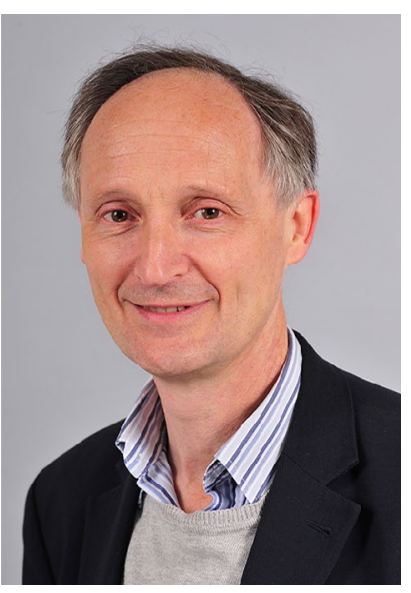

Simon Donell
The patellofemoral joint is enigmatic. The symptoms that arise from pathology of the extensor mechanism are mainly anterior knee pain and instability. However, in many patients the pain felt at the front of the knee is referred and does not arise from the joint. In addition, many patients have an unstable extensor mechanism, not because the mechanism is structurally abnormal, but because of poor muscle control.

Simon Donell

simon.donell@nnuh.nhs.uk

Lars Blønd

lars-blond@dadlnet.dk

1 Koege University Hospital and Teres Medical Group, Copenhagen, Denmark

2 Norfolk and Norwich University Hospital, Colney Lane, Colney, Norwich NR4 7UY, UK
Orthopaedic surgeons like operating. We operate to correct anatomical abnormalities and correct pathology. It is therefore entirely logical to argue that abnormal articular cartilage should be repaired or replaced. It is also logical that we should try new treatments to see whether we can find a suitable method for repairing abnormal articular cartilage. This raises the question, what is abnormal articular cartilage? For many years, soft articular cartilage in the patella has been given a disease status and called chondromalacia patellae. Two decades ago, this was the diagnosis in adolescents presenting with anterior knee pain. The term is still in use for this age group and is included in a "syndrome" patellofemoral pain syndrome, even though it has none of the features of a syndrome. "A medical syndrome is a specific collection of signs, symptoms, investigation results that occur together often enough, so that the presence of one feature indicates the presence of the others. The causes of a syndrome can be investigated, as can its management and even its cure (consider hypermobility or complex regional pain syndromes)" [7]. Nowadays "chondromalacia patellae" has reappeared in MRI scan reports where the finding correlates with increasing age. Many patients with anterior knee pain over the age of 30 years old now are sent with MRI scans that report chondromalacia patellae as if this is the source of their pain. However, it is important to understand that the images produced by magnetic resonance technology are similar to the images seen by the prisoners in Plato's Cave; they are shadows of reality [13]. They are not the patient, and they are not the patients' knee. They are the interpretation of anatomy and pathology based on the behaviour of atoms. Articular cartilage does not contain nerves and in itself cannot experience pain. The real problem is that over the age of 30 years, we begin to age. Articular cartilage is a bearing surface and wears with age. Although we know that changes in articular cartilage 
that lead to "osteoarthritis" start years before the onset of symptoms, we do not know the difference between ageing articular cartilage and osteoarthritic articular cartilage, especially when looking at radiographs. On top of this, all musculoskeletal tissues also show age-related changes, including the muscles.

We also need to consider that $30 \%$ of patients over the age of 50 years present with changes that are described as "osteoarthritis" and yet the majority do not experience pain. Yet pain is an absolute requirement for the diagnosis of osteoarthritis. We can therefore conclude that changes seen on a radiograph which show degeneration are not in themselves the cause of any pain experienced. Between us we have now operated on seven patients who have presented with trochlear dysplasia and exposed bone on both sides of the patellofemoral joint. They have undergone an "excision" trochleoplasty where the trochlea was reshaped to form a groove leaving the cancellous bone exposed and un-resurfaced. The patella may need to have been reshaped as well to create congruency, and the extensor mechanism realigned to get the patella to track normally. These patients have recovered without significant problems. One patient, aged 39 years, described his knee as "normal" 3 years later. This included playing cricket as a wicket keeper, which requires a squatting stance and jumping from this position. None of these patients have normal articular cartilage, but they do have satisfactory patellofemoral kinematics.

It is known that younger patients who experience anterior knee pain (AKP) are more likely to have trochlear dysplasia, compared to pain-free individuals [14]. Keser et al. [11] observed that the lateral trochlear inclination angle, which is associated with trochlear dysplasia, was significantly lower in subjects with AKP compared to patient without. A reduced lateral trochlear inclination angle is a finding that has also been closely associated with patellofemoral instability [2]. So how could trochlear dysplasia induce the pain? Biedert et al. [1] have found that one of the characteristics of the dysplasia is that the trochlear height is significantly higher compared to normal knees; the trochlear boss or bump. The patella that tracks over the boss raises the joint reaction force. In patients with a trochlear boss and AKP, there is an elevated hydrostatic pressure and also increased water content in the patella [8]. In another study comparing AKP patient with normal individuals, there is a significant higher post-exercise chondral thinning in the latter as measured by MRI scan [6,9]. Draper et al. [4] measured increased metabolic activity in the patella and trochlea area using PET/CT scans and found that the tracer uptake correlated significantly with the level of pain. Van Haver et al. [16] found that in a simulated trochlear dysplasia cadaver model, there was increased pressure in the PF joint. It is also interesting to note that Kalichman et al. [10], Mofidy et al. [12], and Stefanik et al. [15] have all found a relationship between low lateral trochlear inclination angle (trochlear dysplasia) and cartilage wear or PFJ arthritis. Dye [5] has said that increased cartilage wear leads to synovitis and that leads to pain. There is a rich nerve supply in the complex synovial folds around the PFJ [3] which may also be stimulated by abnormal movements of the patella if there is maltracking or poor muscle control. If trochlear hyperplasia leads to increased pressure in the PFJ, then, logically, removing the bump with a deepening trochleoplasty will improve PFJ congruence and establish smooth kinematics. This will then reduce the pain. This is more important than creating "normal" articular cartilage where it is damaged.

Therefore does the patellofemoral joint really need articular cartilage? If it is worn away, does it really need replacing? Given that we have patients without patellofemoral articular cartilage who are pain free following surgery, it seems logical to conclude that, in the patellofemoral joint, congruency and smooth kinematics are more important than "normal" articular cartilage.

\section{References}

1. Biedert RM, Bachmann M (2009) Anterior-posterior trochlear measurements of normal and dysplastic trochlea by axial magnetic resonance imaging. Knee Surg Sport Traumatol Arthrosc 17(10):1225-1230

2. Carrillon Y, Abidi H, Dejour D, Fantino O, Moyen B, TranMinh V (2000) Patellar instability: assessment on MR images by measuring the lateral trochlear inclination-initial experience. Radiology 216(2):582-585

3. Donell ST (1992) The synovial folds of the patellofemoral joint: a dynamic study. Clin Anat 5:107-112

4. Draper CE, Fredericson M, Gold GE, Besier TF, Delp SL, Beaupre GS et al (2012) Metabolic activity at the patellofemoral joint. J Orthop Res 30(2):209-213

5. Dye SF (2005) The pathophysiology of patellofemoral pain: a tissue homeostasis perspective. Clin Orthop Relat Res 436:100-110

6. Farrokhi S, Colletti PM, Powers CM (2011) Differences in patellar cartilage thickness, transverse relaxation time, and deformational behavior. A comparison of young women with and without patellofemoral pain shawn. Am J Sport Med 39(2):384-391

7. Grelsamer R, Moss G, Ee G, Donell S (2009) The patellofemoral pain syndrome; the same problem as the Loch Ness Monster? Knee 16:301-302

8. Ho KY, Hu HH, Colletti PM, Powers CM (2014) Recreational runners with patellofemoral pain exhibit elevated patella water content. Magn Reson Imaging 32(7):965-968

9. Ho KY, Keyak JH, Powers CM (2014) Comparison of patella bone strain between females with and without patellofemoral pain: a finite element analysis study. J Biomech 47(1):230-236

10. Kalichman L, Zhang Y, Niu J, Goggins J, Gale D, Felson DT et al (2007) The association between patellar alignment and patellofemoral joint osteoarthritis features: an MRI study. Rheumatology 46(8):1303-1308

11. Keser S, Savranlar A, Bayar A, Ege A, Turhan E (2008) Is there a relationship between anterior knee pain and femoral trochlear dysplasia? Assessment of lateral trochlear inclination by magnetic resonance imaging. Knee Surg Sport Traumatol Arthrosc 16(10):911-915 
12. Mofidy A, Veravalli K, Jinnah RH, Poehling GG (2014) Association and impact of patellofemoral dysplasia on patellofemoral arthropathy and arthroplasty. Knee 21:509-513

13. Plato. The Republic. (Translated by Benjamin Jowett) The internet classics archive. http://classics.mit.edu//Plato/republic.html. Accessed 16 June 2015

14. Powers CM (2000) Patellar kinematics, part II: the influence of the depth of the trochlear groove in subjects with and without patellofemoral pain. Phys Ther 80(10):965-978
15. Stefanik JJ, Roemer FW, Zumwalt AC, Zhu Y, Gross KD, Lynch JA et al (2012) Association between measures of trochlear morphology and structural features of patellofemoral joint osteoarthritis on MRI: the MOST study. J Orthop Res 30(1):1-8

16. Van Haver A, De Roo K, De Beule M, Labey L, De Baets P, Dejour D et al (2014) The effect of trochlear dysplasia on patellofemoral biomechanics: a cadaveric study with simulated trochlear deformities. Am J Sport Med 43(6):1354-1361 